\title{
YouTeach-An E-Learning Portal
}

\author{
Naitik Shah \\ K. J. Somaiya \\ College of \\ Engineering. \\ Mumbai-400077
}

\author{
Payal Mehta \\ K. J. Somaiya \\ College 0 \\ Engineering. \\ Mumbai-400077
}

\author{
Piyush \\ Vishwakarma \\ K. J. Somaiya \\ College of \\ Engineering. \\ Mumbai-400077
}

\author{
Deepak Sharma \\ Associate Professor \\ K. J. Somaiya \\ College of \\ Engineering \\ Mumbai-400077
}

\begin{abstract}
The acquisition of knowledge along with its use facilitated and distributed by electronic means is called as E-Learning. E-learning can take the form of smaller learning objects or courses as well as modules. The physical barriers of learning are eliminated and have enhanced indigenous participation in education. E-learning also allowed flexible learning opportunities. A lot of resources are needed by an individual to gain an expertise in a particular domain. The knowledge required to gain expertise is now easily available due to the advent of E-learning. The resources on the internet are growing at a very fast rate. A secure and efficient system is needed so that users can be directed to area of his interest such that he can learn and gain insights in the domain. Development of an organized learning atmosphere will help the user to find the required information at a same location. ELearning has been identified as positive, interesting and creative way for indigenous students to learn by the literature. This paper presents Youteach an E-Learning portal which focuses on providing an accessible and user friendly interface to the students to learn any educational concepts based on varied fields. The portal will be made with the capability of generating multimedia content (video or files) which will be uploaded by distinguished professors of the college or competent users and the content could be on varied educational topics.
\end{abstract}

\section{General Terms}

Cloud Computing, Content Management System

\section{Keywords}

E-Learning portal

\section{INTRODUCTION}

The paper is based on providing an E-Learning experience to the users. It is mainly focused to provide the users with a new day technological educational means by adding up on the conventional learning education system. The amount of ELearning material is growing on the internet day in and day out. A proper system is required that could arrange this worldwide knowledge as per users need and benefits. Digitization has come a long way to boost users to move to ELearning systems. E-Learning systems provide users with major benefits like to learn a topic at their own speed, to learn them based on their schedule and get the best quality of information and resources to learn from. Moreover, an ELearning platform also helps a person to share his view and expertise on the topic of his interest. The Youteach is an integration of various existing E-Learning websites; social websites and user needs for simpler means of education. Youteach platform is an internet based platform which is available far and wide.

Youteach tries to devise a platform where a student can learn or teach via internet. Distance learning programs are the roots for the advancements of E-learning experience via the internet connections across the world. Various forms of electronic media are now used to provide the best learning and teaching to students via the usages of projector screens, presentations, intranets, learning management systems or internet video streaming. E-Learning can thus be defined as the knowledge that is shared beyond the physical frontiers that covers distance via the usage of electronic media or internet. An ELearning system is basically divided into Learning Management System, technology used to build the Learning Management System, the continual maintenance of the LMS built and the contents that show the course information over the platform. The LMS on the Youteach platform is based on a PHP bootstrap framework along with storage on cloud. Youteach will provide an organized learning mechanism to all its users with varied resources over the courseware. A The project presents a building up of a useful LMS on cloud. Researches based on E-Learning with respect to learners and teachers using such platforms will help to further develop the framework of Youteach. The detailed description of the project structure is cited in the paper, along with the related theory and new advancements in the Youteach platform. [3]

\section{RELATED WORK}

The previous citations and websites have attempted to solve the problems related to E-Learning and have been very successful in this field. There are various e-learning systems which provide courseware to users across the world. These systems have renowned experts to teach on various topics. They have a scheduled courseware and students can register for it either free or with some charges. The users are then provided with the certificate of accomplishment post the completion of the course. NPTEL [5] is one such E-Learning system that provides video courses across various streams mainly in engineering disciplines. This system was initiated by IITs and IISc Bangalore in India and now is used across the world. Each of the courses consists of about 40 video lectures of approximately an hour each. This system has a decent interface and video management system. But the amount of response time is an issue. In case of unavailability of high speed internet, the system loading takes a huge amount of time for loading. Moreover it is a one-way system where there is no facility of interaction by the students to the professor directly. Only way to get connected is via feedbacks. Also there are no test series available for checking whether the student has understood the topic. Only the professors are allowed to upload the courses and the user credentials are not sored in this system. So overall it is only knowledge based E-Learning system. Another such system popularly known as Coursera[4] which is an educational technology company which offers extensive range of courses from various disciplines like Mathematics, Physics, Biology, Engineering, and Business and so on. It has tie ups with certain top organizations and universities which provide the courses on this system with their authorization. Coursera provides a proctored exam at the end of a particular course 
named ProctorU. They also provide quizzes and interactive session during the course work. The services provided by the company are too expensive and only specific experts have the authority to upload courses. Anyone having an expertise or some research in a particular field is usually not directly allowed to upload the courses.

\section{PROPOSED SYSTEM}

Youteach is developed in iterations following the spiral-model approach. In the initial iterations, the prototype is built showing all the basic functionalities of the website. The project will be requiring a web platform for the web based portal using Bootstrap along with backend indexing and using Cloud computing for data storage and faster retrieval. The web based portal will be a complete website consisting of the login page, the registration page and pages to upload, download or view courses, browse the courses, and post queries (in discussion forum). The courses that will be posted must be verifies by a moderator before they are published online. This portal will be developed by the web developers and designers of the organization using CodeIgniter [8] as the bedrock using the code igniter's model view controller (MVC) framework. The model framework in the Youteach website is used to represent all the data structures that are used and will assist to insert, retrieve and update all the information in the Youteach database. The view framework shows the information displayed to the end user, that is, the user interface as a web page. The controller framework acts as an intermediary between the model and the view and all other resources that are used in the Youteach website. Various APIs have been implemented to develop some of the modules as per the required functionalities. All the modules will be given in detail in the next section of the paper.

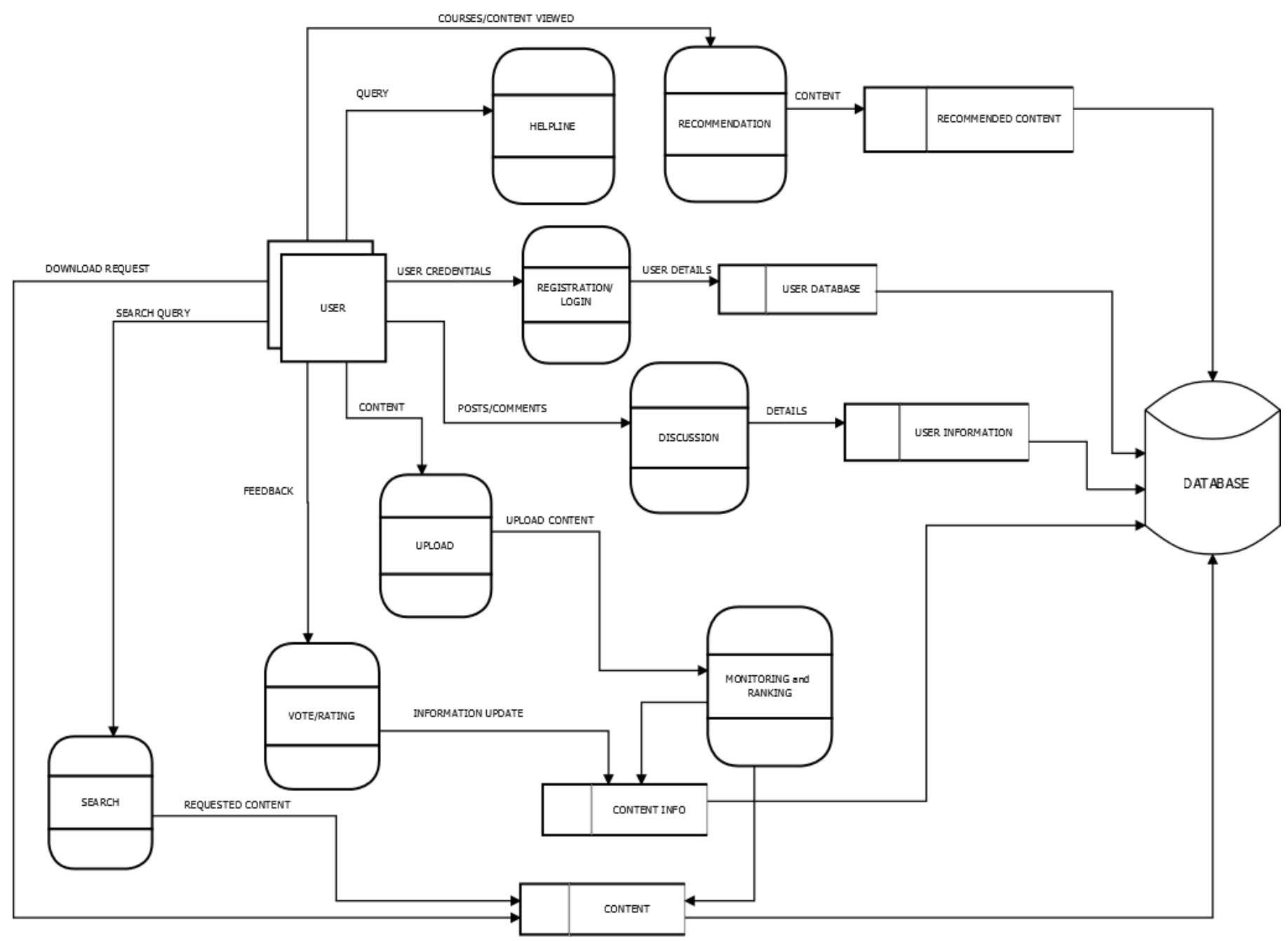

Fig 1: Architecture of system

\section{METHODOLOGY AND IMPLEMENTATION}

The Youteach project is developed based on spiral model using various modules each having specific functionalities. The basic module are: the registration/user login module where a new user registers himself to access Youteach completely and logs in each time he wants to resume his incomplete work or start a new course. Also, the Youteach portal will include a set of guidelines to assist its new users and get them acquainted to the YouTeach interface.

The system YouTeach is deployed on the AWS cloud. The steps mentioned in this link are referred for the same. [10] 


\section{- Indexing and Searching Module}

For indexing, searching and auto-completion module, we have used OpenSearchServer [1], which is a java based standalone server.

The main features which are extended by this plugin architecture for YouTeach are:

- Textual search.

- A crawler to index the following: Content from databases (MySQL).

- Full text analyzers and filters allowing indexing and searches.

- An HTML renderer allowing the integration of the search box in an html page, working with PHP over http API.

The following steps are followed to crawl a database:

1. First, we created a database with the required tables and fields.

2. Then we created an index that would be used to index the data.

3. In order to transform the data, analyzers were decided upon and a regular expression was entered to match with the data and return appropriate results.

This analyzer will be used to extract the year and the month from a full date. This data will then be used as a facet to filter the content from the database according to timestamp added.

4. The following schema was then defined:

5. In this step, we actually worked with the database created in the first step.

We set a crawler on the database by using the following settings:

To map the settings, the following changes were made in the field map tab:

6. The last step done was to search a query. An HTML renderer was created to display the results to the user and CSS was used to represent the data in a desirable format.

\section{- Recommendation Module}

Since, the web is a massive collection of data and finding content according to one's preferences is a time consuming activity for the user, we have implemented a rating-based item-to-item recommender system in PHP/ SQL to simplify the visual search for content of a user. In this approach, we have taken into consideration the ratings provided by the user for providing non-personalized recommendations and the user's profile for providing personalized recommendations. It is assumed that the user would rate items on a scale of 1 to 5 .

From the database point of view, we have created two tables, a "rating" table with the following attributes userID, itemID, ratingValue, timestamp for storing the rating records and a "dev" table which would be updated as and when a user rates an item. This table contains the following attributes: itemID1, itemID2, count, and sum. Suppose there are about 20 users who have rated both item 1 and item 2 . We sum the ratings obtained by both the items. Suppose item 1 has obtained 55 ratings and item2, 75 ratings. Clearly, item 2 is rated higher than item 1 by 1 on average. ((75-55)/20). For updating the "dev" table, the code has been cited. [2]

Using the above mentioned approach, we have implemented an item-to-item based recommendation system. This approach does provide sensible recommendations and is quite easy to implement.

\section{- Moderator Module}

The major concern of a video sharing website is monitoring the authenticity, quality, uniqueness \& usefulness of the uploaded content. Youteach will consist of skilled professionals, known as "Moderators" who will have rights to check on all uploaded contents as well as the user activities. Moderators will act as the human firewalls who will prevent any direct access to important data manipulation (like course/documents upload, etc.) in the system by the users. Following are the major functions, a moderator can perform.

\section{- Promotion to a teacher}

To prevent the site from flooding of fake or invalid teacher accounts, a moderator will decide whether to give course uploading rights to a person or not. Apart from system inbuilt CAPTCHA for human verification, moderator will check scanned identity proof along with all other details submitted.

\section{- Approval of course}

The moderator will decide whether a particular course is suitable for publishing or not. For any new course that a user wants to upload, a request along with the course information and the video files will be first made available to the moderator who will approve it and then made available online for all other users. Person who will check or approve a given course will be specialized or will be having some knowledge about the given course domain.

\section{- Managing Users and their activities}

Profiles of all users, including their recent and later activities will be made available to the moderator. $\mathrm{He} / \mathrm{sh}$ can blacklist users or block their accounts in case of any unusual activity (like spamming, use of abusive language or flooding the site with harmful/not-useful data) detected.

\section{- Course editing/removing rights}

Once after finalized and made online, the Teacher cannot make changes or delete the courses without prior approval from the moderator. He needs to summarize briefly about the changes or why he/she wants to remove a particular course uploaded by him/her and seek a request for editing or removing a course. The moderator will reply positively for may deny this request depending on the situation.

\section{- Course archiving and blocking}

Moderator can archive or block a particular course, depending on various situations as when needed.

\section{- Content upload/download module}

Content upload/download is made available to all the users but can be used only based on specific steps.

a) Content Upload: Uploads in Youteach are based on JQuery-file-uploads [6] which has cross-browser compatibility by using XMLHttpRequest objects in browser and the FileReader. This eventually supports an iframe Transport module for all the 
browsers which are incompatible with XHR file uploads. The client side code consists of an extended jQuery which is highly helpful. The server side consists of PHP, GAE-Go and Google App engine (GAE) for Python. Two servers that are used - WAMP and Sambar both support PHP completely. The plugin is divided into two major parts: the first basic plugin is used to implement the uploading of file process as already described, while the UI version adds advanced functionality such as server-side image rescaling for thumbnail previewing and a complete User Interface. The text page is based on Phil Parsons Ajax upload with the File API blog post and XMLHttpRequest level. Multiple file selection is allowed at the same time which includes information display and provides previews for images. The file selection is done using a file input control.[7]

b) Content download and viewing: All the registered users will be allowed to view the rendered course online and download it for their reference. The data is stored over the cloud so the downloading of videos, text, image and other content will be accessible and fast which would ease the life of the user. [9]

\section{- Discussion Module}

Of all the content mentioned in the Youteach website, there will be a discussion forum which will help the users to directly interact with other students who are learning the course in case of any queries. A user can also get connected to the professor directly by posting queries on certain topics. The discussion module will help users to better interact with all others. Also the user can ask for extra study materials on a topic from the tutor.

\section{RESULTS}

The Homepage of you-teach.in consists of a simple user interface for the user

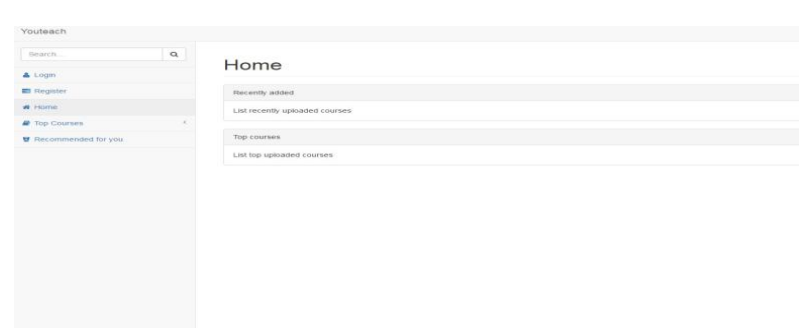

Fig. 2 Homepage of you-teach.in

A user expert in a particular domain can upload a course.

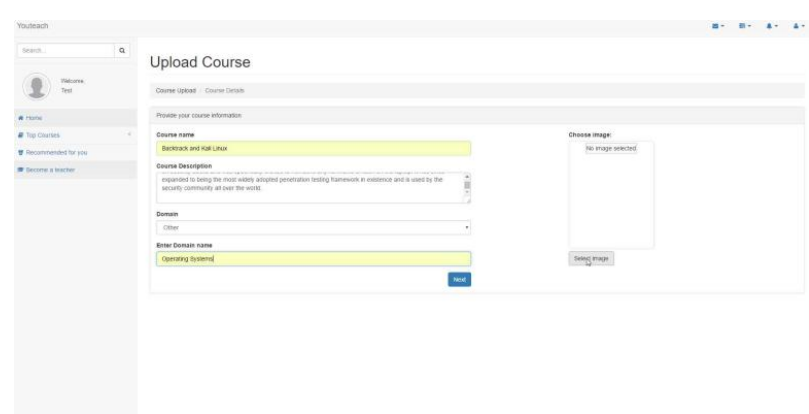

Fig. 3 Uploading Course Details
The contents like videos and associated documents can be uploaded by the user.

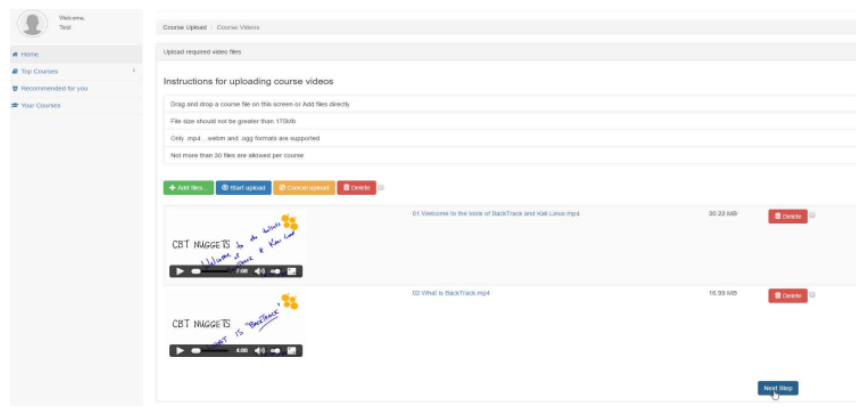

Fig. 4 Uploading Course Contents

The moderator approves the course at the backend after authenticating it.

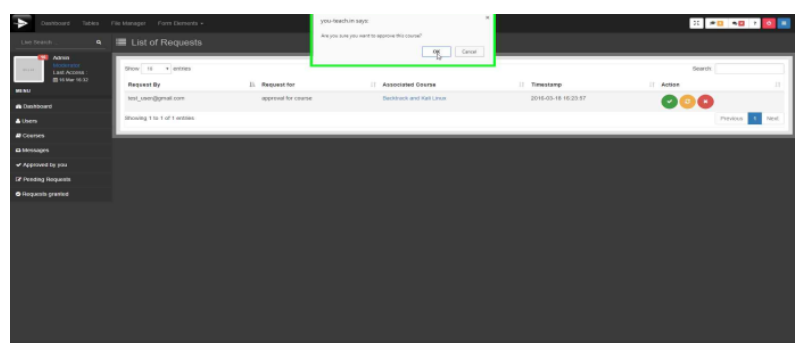

Fig. 5 Course Approval

The course once live on you-teach.in has the columns to rate, comment,navigate a course, and access the related documents.

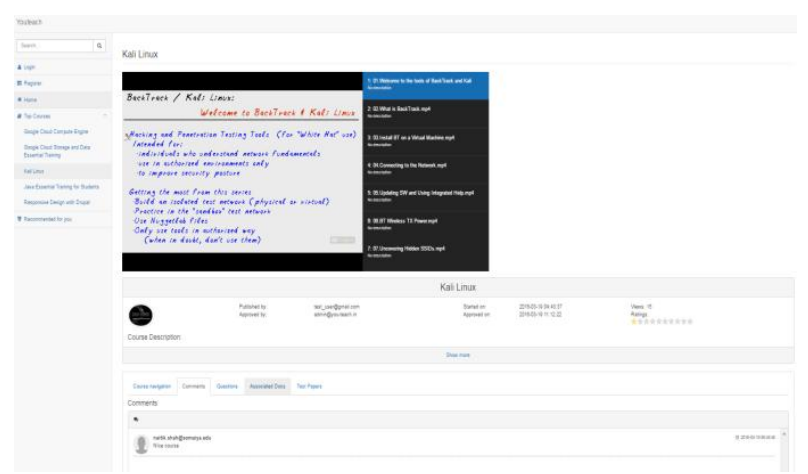

Fig. 6: Course Display

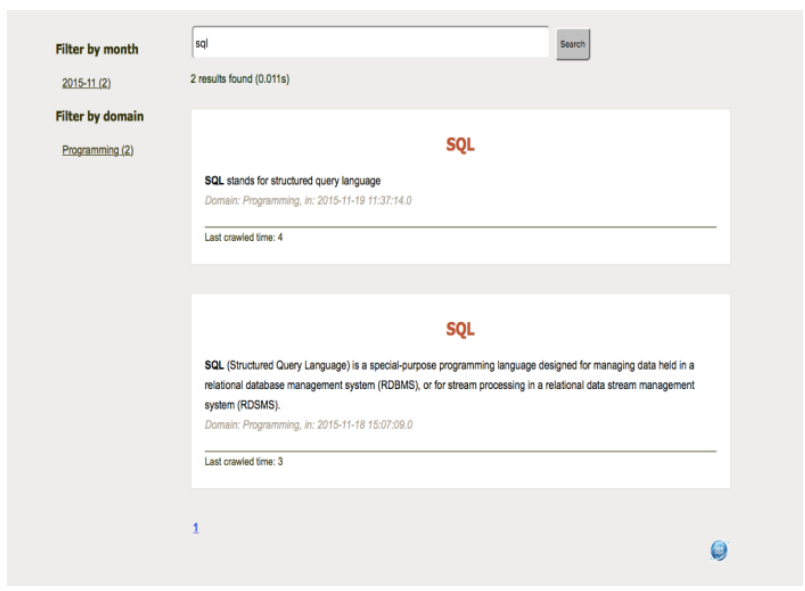

Fig. 7: Keyword searching 
Table 1: Comparisons with Existing System based on features

\begin{tabular}{|c|c|c|c|c|}
\hline Existing Systems & Interactive & Moderator System & User Contribution & Recommendations Provided \\
\hline Coursera & Yes & No & No & No \\
\hline NPTEL & No & No & Yes & Yes \\
\hline $\begin{array}{l}\text { Our Approach } \\
\text { (you-teach.in) }\end{array}$ & Yes & Yes & \\
\hline
\end{tabular}

\section{FUTURE SCOPE}

The Youteach website can create a unique experience to the users and can provide excellent platform to users to learn as well as showcase their talent by uploading a course in their domain of interest. In future, the Test module can be added to the Youteach website which can access the user's ability based on the course they have learnt. Also based on the credits, a statement of accomplishment can be provided to the user to keep him encouraged to learn more courses and enhance his skillset. Interactive systems can be built in future to assist the teachers in the colleges or school to interrogate the students online, thus reducing the amount of time required and simplifying the job of the teachers. A scholarship search engine can be integrated with the Youteach in future to match the user's ability with the best knowledge they can receive across the world. Scholarships can be awarded to students to pursue their studies or startups. Thus the scope goes on increasing, and it will be forever facilitating the conventional methods of education and striving to give the world the best knowledge possible.

\section{CONCLUSION}

Usually an E-Learning portal allows users to learn the courses but doesn't allow them to actually upload it. Youteach provides the functionality to users to show their expertise. Youteach is thus a platform for both students to get expertise and other users to show their research. The project presents Youteach E-Learning portal's concept, its development blocks and future scope in this field. The paper presents a conceptual model along with its initial implementation to devise a system to provide knowledge through internet.

\section{ACKNOWLEDGMENTS}

The authors would like to thank Mr. Deepak Sharma of K. J. Somaiya College of Engineering for providing specifications and insights on the E-Learning portal.

\section{REFERENCES}

[1] "OpenSearchServer Documentation - Crawling a database", Opensearchserver.com. [Online]. Available: http://www.opensearchserver.com/documentation/tutoria 1s/crawling_a_database.md. [Accessed: 04- Mar- 2016].
[2] D. Lemire and S.McGrath, "Implementing a RatingBased Item-to-Item Recommender System in PHP/SQL", vol. -01, 2013.

[3] T. Kaewkiriya, N.Utakrit and H.Tsuji, "Experimental Evaluation of Distributed e-Learning Management System", IEEE, vol. 978-1-4577-0255-6, 2011.

[4] "Coursera", Wikipedia [Online]. Available: https://en.wikipedia.org/wiki/Coursera.[Accessed: 03Mar- 2016].

[5] "National Programme on Technology Enhanced Learning", Wikipedia [Online]. Available: https://en.wikipedia.org/wiki/National_Programme_on_T echnology_Enhanced_Learning. [Accessed: 03- Mar2016].

[6] "jQuery File Upload Demo", Blueimp.github.io [Online]. Available: https://blueimp.github.io/jQuery-File-Upload/ [Accessed: 02- Jan- 2016].

[7] "Using HTML5 and the Blueimp jQuery-File-Upload Plugin To Upload Large Files", Htmlgoodies.com.[Online]. Available: http://www.htmlgoodies.com/html5/other/using-html5and-the-blueimp-jquery-file-upload-plugin-to-uploadlarge-files.html\#fbid=fnvoJfJfeam.[Accessed: 01- Jan2016].

[8] I. EllisLab, "CodeIgniter /A EllisLab", Ellislab.com [Online].

Available: https://ellislab.com/codeigniter.[Accessed: 03- Aug2015].

[9] "Render images, sound and videos inside Editor", DataTables forums [Online]. Available: https://datatables.net/forums/discussion/19859/renderimages-sound-and-videos-inside-editor.[Accessed: 04Mar- 2016].

[10] "Tutorial: Installing a LAMP Web Server on Amazon Linux - Amazon Elastic Compute Cloud", Docs.aws.amazon.com [Online]. Available: http://docs.aws.amazon.com/AWSEC2/latest/UserGuide/ install-LAMP.html.[Accessed: 01- Jan- 2016]. 